\title{
Mitarbeiter der Generation Y führen
}

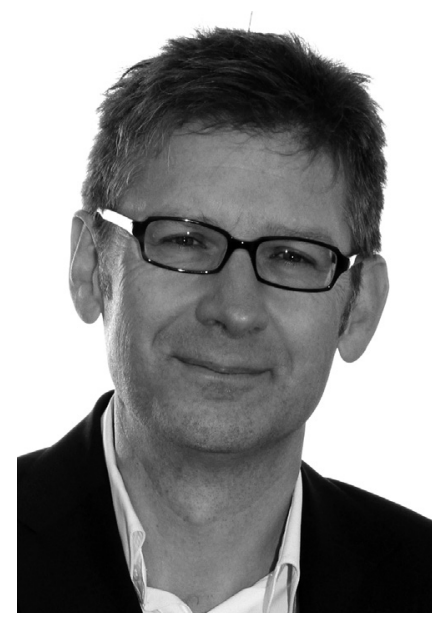

VON HUBERT HÖLZL

Hubert Hölzl ist Inhaber des Trainings- und Beratungsunternehmens Hölzl \& Partner in Lindau. www.fuehrungstrainer.net

\author{
Die nachrückenden Leistungsträger in Organisationen \\ und Unternehmen reagieren oft anders als \\ ihre älteren Kollegen. Deshalb muss sich der \\ Führungsstil in den Betrieben wandeln.
}

In unserem Unternehmen muss sich ein fundamentaler Wandel im Bereich Führung vollziehen. Dieses Gefühl plagt viele Verantwortliche auch in der Sozialwirtschaft. Denn immer mehr Indikatoren deuten darauf hin, dass die traditionelle Art zu führen, an ihre Grenzen stößt. Beispiele: die sinkende Loyalität der Mitarbeiter; die steigende Zahl der Führungskräfte, die über eine Überlastung klagen; die wachsenden Probleme vieler Unternehmen, den Wandel zu meistern.

All diese Faktoren tragen dazu bei, dass sich bei den Entscheidern das Gefühl verdichtet: In unserer Organisation muss sich ein grundlegender Wandel in der Führung vollziehen. Sonst kann unser Unternehmen, die Herausforderungen, vor denen es steht, mittelfristig nicht mehr meistern.

Die Ursachen hierfür sind vielfältig. So stehen heute die Unternehmen zum Beispiel unter einem weit höheren Veränderungsdruck als früher - woraus auch eine permanente Mehrbelastung der gesamten Organisation resultiert. Zudem haben sich im zurückliegenden Jahrzehnt in den meisten Unternehmen neben den Arbeitsinhalten die Arbeitsstrukturen und Arbeitsbeziehungen verändert. Was sich jedoch kaum geändert hat, ist die Art, Mitarbeiter zu führen.

\section{Auch die Mitarbeiter haben sich verändert}

Ein solcher Wandel wäre jedoch dringend nötig - nicht nur, weil es den Unternehmen aufgrund des demographischen Wandels zunehmend schwer fällt, ihren Bedarf an qualifizierten Mitarbei- ter zu decken. Entscheidender ist: Die Mitarbeiter haben sich verändert.

In den Unternehmen rückt mit der

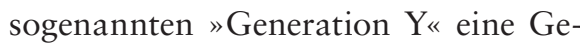
neration junger Frauen und Männer nach, die ein anderes Wertesystem als deren bisherige Leistungsträger haben. Sie sagen zwar auch Ja zur Leistung, betrachten Arbeit aber primär als ein Instrument zur Sicherung der Existenz und des gewünschten Lebensstandards. Und zudem wollen sie ihre Arbeit als befriedigend erfahren.

Das stellt die Führungsmodelle vieler Unternehmen in Frage. Denn in ihnen wird zwar oft ein kooperativer Führungsstil propagiert, die Führungsrealität sieht aber anders aus. In vielen Unternehmen legitimiert Führung ihre Autorität noch primär über die hierarchische Position; des Weiteren über einen Vorsprung an Erfahrung, Wissen und Information. Und häufig wird von den »Untergebenen« im Arbeitsalltag vor allem Gehorsam erwartet.

Und gute Führung? Sie wird daran gemessen, wie die Mitarbeiter »spuren«. Zugleich wird aber betont: Unsere Mitarbeiter müssen eigenständiger denken und handeln. Hieraus resultiert ein Grundkonflikt, für den viele Unternehmen noch keine Lösung gefunden haben.

\section{Aus Mitarbeitern werden Kooperationspartner}

Generell gilt: Im Zeitalter unternehmensübergreifender Projekte und Netzwerke sowie fließender Strukturen lassen sich die Grenzen zwischen Führenden und Geführten nicht mehr so klar 


\section{Was versteht man eigentlich unter der "Generation Yu?}

Als "Generation Y" wird in der Soziologie diejenige Bevölkerungskohorte genannt, deren Mitglieder um das Jahr 2000 herum zu den Teenagern zählten. Je nach Quelle wird diese Generation auch als "Millennials" (zu deutsch etwa "die Jahrtausender«) bezeichnet. Sie gilt damit als Nachfolgegeneration der Baby-Boomer und der Generation X. Die Generation Y gilt als vergleichsweise gut ausgebildet, oft mit Fachhochschul- oder Universitätsabschluss. Sie zeichnet sich durch eine technologieaffine Lebensweise aus, da es sich um die erste Generation handelt, die größtenteils in einem Umfeld von Internet und mobiler Kommunikation aufgewachsen ist. Sie arbeitet lieber in virtuellen Teams

wie früher ziehen. Die Führungskräfte verlieren an Einfluss und den Mitarbeitern fällt eine aktivere Rolle zu. Und zunehmend entscheidet der Grad der Kooperation darüber, wie innovativ und leistungsstark ein Unternehmen ist.

Das erfordert eine Führung, die unabhängige Leistungsträger unter einem gemeinsamen Ziel vernetzt kann. Hierfür benötigen Führungskräfte die Akzeptanz ihrer Mitarbeiter. Denn sie entscheiden zunehmend selbst, ob sie einer Person das Recht einräumen, sie zu führen. Und ihr »Ja« zur Führung? Es zeigt sich ihrer Initiative, ihrem Engagement und ihrer Loyalität.

Das ist vielen Führungskräften nicht ausreichend bewusst. Sie erwarten von ihren Mitarbeiter insgeheim noch immer, dass sie sich bedingungslos den Vorgaben ihrer Vorgesetzten und den Zielen des Unternehmens unterordnen. Die Zeiten eines bedingungslosen Gehorsams und einer bedingungslosen Loyalität sind jedoch vorbei. Die Leistungsträger in der nachrückenden Generation wollen außer einem guten Einkommen auch Mitsprache- und Entscheidungsmöglichkeiten sowie Gestaltungsspielräume haben.

Und dies zu Recht! Denn von ihnen wird erwartet, dass sie - sei es alleine oder im Team - ihre Aufgaben weitgehend eigenverantwortlich lösen. Und wenn sich die Rahmenbedingungen ändern? Dann sollen sie eigeninitiativ neue Problemlösungen suchen.

Hierzu sind sie in der Regel bereit. Sofern sie für diese Leistung seitens ihrer Vorgesetzen auch die erforderliche Anerkennung und Wertschätzung erfahren und zwar nicht nur in Form von Phrasen als in tiefen Hierarchien. Anstelle von Status und Prestige rücken die Freude an der Arbeit sowie die Sinnsuche ins Zentrum. Der Berliner Jugendforscher Klaus Hurrelmann macht auf die Multioptionsgesellschaft und Grenzlosigkeit aufmerksam, in welcher die Generation Y groß geworden ist. Die Millennials sind optimistisch und selbstbewusst und haben wenig Vertrauen in die Regierung, weshalb sie sich durch passiven Widerstand aktiv ins politische Geschehen einbringen. Ein Beispiel dafür ist die Bewegung Occupy Wall Street, wodurch sich die moderne Organisation der Generation Y abzeichnete.

Quelle: http://de.wikipedia.org

wie "Das schaffen Sie schon « oder "Das haben Sie gut gemacht«. Sie erwarten von ihren Führungskräften vielmehr eine aktive Unterstützung, Weitergabe von Information und Einbindung in Entscheidungsprozesse.

\section{Sich wechselseitig vertrauen und ernst nehmen}

Dahinter steckt eine weitere Erwartung der Leistungsträger von morgen. Sie wollen das Gefühl haben: Ich und das, was ich tue, haben eine Bedeutung. Sie lassen sich nicht mehr mit Motivationstricks aus der Führungsmottenkiste stimulieren. Sie wollen ihre Arbeit selbst als sinnhaft erfahren. Gelingt ihren Führungskräften diese Sinnvermittlung nicht, sinken ihr Commitment und Engagement. Zudem steigt die Fluktuation. Denn die Leistungsträger von morgen sind nicht nur anspruchsvoller als ihre Vorgänger, sie ist auch unabhängiger. Erfüllt ein Arbeitgeber ihre Erwartungen nicht, wechseln sie zum Wettbewerber.

Deshalb ist im Bereich Führung in vielen Unternehmen ein Musterwechsel nötig. Ihre Führungskräfte müssen künftig, um Talente zu binden, eine vitale Kooperationsbeziehung mit ihnen eingehen - auf Augenhöhe. Dies setzt ein wechselseitiges Vertrauen und Ernstnehmen voraus, das sich im tagtäglichen Miteinander dokumentiert. Das erfordert wiederum, dass die Führungskräfte nicht nur von ihren Mitarbeitern fordern, ihr Denken und Handeln zu reflektieren und bei Bedarf zu ändern. Auch die Führungskräfte selbst müssen ihr (Führungs-) Verhalten hinterfragen und gegebenenfalls revidieren, so dass ihre Performance als Führungskraft steigt. Oder anders formuliert: Sie müssen sich auch selbst - und nicht nur ihre Mitarbeiter - als Lernende begreifen.

\section{Auch die Führungskräfte müssen lernen}

Führungskräfte neigen dazu, den eigenen Anteil am Verhalten der Mitarbeiter zu negieren. Mehr noch: Sie kommen oft gar nicht auf die Idee, dass dieses etwas mit ihnen selbst zu tun haben könnte. Entsprechend selten fragen sie sich zum Beispiel: Was habe ich unternommen, dass sich mein Mitarbeiter anders als von mir (oder vom Unternehmen) erwartet verhält? Oder: Zeigen meine Mitarbeiter so wenig Eigeninitiative, weil ich sie weitgehend mit Anweisungen führe?

Das heißt: Viele Führungskräfte sind sich der Wechselwirkung von Kommunikation nicht ausreichend bewusst. Dieses Bewusstsein gilt es zu fördern, damit in der Organisation eine Kultur der Selbstverantwortung und wechselseitigen Kooperation entstehen. Eine solche Führungskultur zu entwickeln, ist eine nicht delegierbare Managementaufgabe. Denn sie entscheidet künftig weitgehend über den Erfolg von Unternehmen. 